\title{
Storage Management and Wastage of Reproductive Health Medicines in Public Health Facilities of West Wollega Zone, Ethiopia: Mixed Study
}

Oliyad Kebede ( $\square$ oliyadkebede@gmail.com)

Mizan Tepi University

Gizachew Tilahun

Jimma University

Desalegn Feyissa

Mizan Tepi University

\section{Research Article}

Keywords: store management, medicines expiration, reproductive health

Posted Date: January 7th, 2021

DOI: https://doi.org/10.21203/rs.3.rs-135580/v1

License: @ (i) This work is licensed under a Creative Commons Attribution 4.0 International License. Read Full License

Version of Record: A version of this preprint was published at BMC Health Services Research on April 1st, 2021. See the published version at https://doi.org/10.1186/s12913-021-06291-w. 


\section{Abstract}

Background: Keeping proper storage conditions at health facilities is important to reduce pharmaceutical wastage caused by environmental factors. The expiration of medicines at the health facilities led to wastage of potentially life-saving drugs and unnecessary expenditure on the disposal of those expired medicines. Therefore, the aim of this study was to assess pharmaceutical stores and wastage of reproductive health medicines due to expiration

Method: A facility-based descriptive cross-sectional quantitative and qualitative study was conducted using a checklist, structured and semi-structured questionnaires.

Results: Among 23 health facilities assessed, 17 (73.91\%) (4(100\%) hospitals and 13(68.42\%) health centers) fulfilled desirable storage conditions. The total value of reproductive health medicines wasted due to expire in surveyed facilities was 357,920.52 ETB (12,323.81 US dollars) and the Percentage of Stock Wasted due to Expiration was $8.04 \%$. Levonorgestrel $0.75 \mathrm{mg}$ tablet contributed highest percentage to expired medicines.

Conclusion: Pharmaceutical stores in hospitals had equipment and furniture, fulfilled desirable storage conditions, whereas, significant number of the health centers' medical store did not comply with the recommended storage conditions. Challenges of store management identified were poor infrastructure, administrative challenges, and shortage of human resource. Stock wasted due to expiration was high and the contributing challenge was non-need-based supply. So Ethiopian pharmaceutical Supply Agency should supply reproductive health medicines based on need and concerned government bodies should avail pharmaceutical store infrastructures, hire professionals, and follow health facilities administrations.

\section{Introduction}

Store management is the management of storehouses and stock information, holding and storage of medicines, and the safe custody and protection of stock. Store is a place where we keep stock in the supply chain. Keeping products in store, guarantees satisfying the future customer demand. Therefore, every business stores a quantity of products for future customers' demand. Store management includes broader activities such as holding; quality control; training of stores staff; and clerical administration of stores operation (1).

Keeping proper storage conditions at health facilities is important to reduce pharmaceutical wastage. Pharmaceutical products stored in pharmacies with good storage facilities maintained their potency. The regulatory authorities and pharmaceutical organizations should emphasize the importance of preserving good storage conditions in the facilitation of health care(2). The management of the store should help the flow of supplies from the source to the end-user in the most reliable and economical way without a significant loss of quality, wastage, or theft(3)

The expiration of medicines at the health facilities is a big concern due to its double burden. Firstly, it leads to wastage of potentially life-saving drugs. On the other hand, it causes unnecessary expenditure on the disposal of those expired medicines(4). It was estimated that about $70 \%$ of funds spent on medicines could be lost or wasted. By improving the basic management, it is possible to reduce these losses significantly. For example, it is possible to reduce medicines lost by expiration by $3 \%$, medicines lost by improper storage by $4 \%(5)$.

Studies conducted in Ethiopia, revealed that the medicine wastage is a persistent problem of public health facilities. Poor storage facilities, improper store utilization, inadequate space, stocking expired medicines with usable products, and poor stock rotation, which led to expiry of medicines. Another study also mentioned; guidelines for the storage and disposal of medicines were not available and not followed at lower levels, the available space was not always well utilized or organized, and the practice of first-to-expire-first-out was followed. It also revealed the problem of expired products at all levels, although did not quantify it. The reproductive health $(\mathrm{RH})$ medicines were emphasized because of their nature supply system. The supply chain system for maternal, neonatal, and child health $(\mathrm{MNCH})$ (which are a major constituent of $\mathrm{RH}$ ) medicines is inconsistent and had not been integrated into the Integrated Pharmaceutical Logistics System (IPLS)(6-8). Since IPLS was thought to increase store management performance and decrease value of expired medicines, being not integrated into IPLS could affect the store management and wastage due to expire. As far as the knowledge of investigators is concerned there is no research conducted on the store management and value of expired medicines in Ethiopia. Therefore, the aim of this study was to evaluate pharmaceutical stores and wastage of reproductive health medicines due to expiration at west Wollega zone. This will be a baseline study for stakeholders and academicians to take corrective action and conduct further studies.

\section{Methods}

\section{Study area, Design, and Sampling technique}

The study was conducted in public hospitals and health centres of west wollega zone, Oromia regional state, Ethiopia. West Wollega had 19 administrative woredas and its capital, Ghimbi, is located $441 \mathrm{Km}$ to the west of Addis Ababa. The study was conducted from 15th July, 2019 to 31 st July, 2019.

Facility based cross sectional descriptive study design was employed. Quantitative result was supported with qualitative result. During data collection period there were five public hospitals and $68 \mathrm{HCs}$. Actively giving services for more than 1 year during data collection period were considered as inclusion criteria. All hospitals were included in this study, except one that did not fulfill inclusion criteria. HCs were sampled using Logistics Assessment indicator Tool (LIAT) recommendation. Accordingly, $15 \%$ of the health centers were included (9). But this sample size was less than the number of woredas. So, to get representative sample for all woredas, one health center was selected by lottery method from each woreda using lottery method. Therefor 23 public health facilities were assessed.

Source population were all public hospitals and health centers of west Wollega zone, all pharmacy professionals working in those health facilities, all transaction recording tools (Model 19 and list of expired medicines) and all reproductive health medicines. Study populations were all hospitals and selected 
health centers, selected reproductive health medicines, all store managers in those facilities, selected pharmacy heads, selected PHCU directors, all hospital medical directors, all supply chain coordinators and selected model 22.

\section{Data collection, data management, and analysis}

Structured questionnaires and checklists adapted from Logistics System Assessment tool (LSAT) and Logistics Indicator Assessment Tool (LIAT) prepared by USAID DELIVER Project were used to collect the necessary data $(9,10)$. The data collectors took notes on field notes while observing warehouses and expired medicines.

The data were gathered through self-administrated questionnaires from store managers, by observation \& physical count of stocks, and review of relevant documents. The stores were assessed by observing stores and filling the checklist, and taking note on the general condition of the store warehouse. We collected the wholesale price of each medicine from invoices. We cleaned the collected data for completeness and consistency. Then, we entered the data into MS Excel 2016 spreadsheet and statistical package for social science (SPSS) version 20 to encode and analyze. The findings were summarized using tables, and figures.

The qualitative data were gathered through in-depth face-to-face interview with key informants and the audio were recorded. The probing and flexible questions were prepared in English and translated into the region's working language, Afaan Oromo. Then, each key informant was interviewed approximately for 15-20 minutes in Afaan Oromo. After data collection, the investigator familiarized with the recorded data by listening repeatedly and taking notes. Then, we translated the result into English. The data were coded in Microsoft word table. Data with similar codes were brought together and organized under themes. The report was produced by quoting KIs' narration under each theme.

\section{Data quality assurance}

Principal investigators gave 2 days trainings for data collectors and strict instructions. The recruited data collectors were pharmacy professionals (druggists and pharmacists). Investigators also supervised the data collectors during data collection. Incomplete and inconsistent questionnaires were taken back to the facility and the data were re-collected.

\section{Ethical Consideration}

This study was conducted in accordance with the declaration of Helsinki. Accordingly, the research was approved by the ethical review board of Jimma University, Institute of Health. Then, the Review Board gave us permission to obtain letter of permission from each facility and verbal consent from all participants. The permission to conduct the study was then obtained from each facility administrations and verbal consent was obtained from all participants. We excluded the name of participants to keep confidentiality.

\section{Measurement of Variables}

The facilities' pharmaceutical warehouses were evaluated using good storage criteria. These criteria were adopted from Ethiopian Pharmaceutical Supply Agency monitoring and evaluation manual and logistics indicator assessment tool $(\operatorname{LIAT})(9,11)$.

Percentage of fulfillment of the storage condition for each facility:

No. of YESresponses * 100

$\overline{\text { Total number of storage conditions considered(17) }}$

After calculating the percentage of fulfillment of the storage condition, those facilities that fulfilled the criteria more than/equal to $80 \%$ were considered as desirable, whereas, those fulfilled less than $80 \%$ were considered undesirable(1).

Percentage of facilities that maintain acceptable storage conditions:

Percentage of facilities that maintain acceptable storage conditions

Total no. of facilities or warehouses visited

Fulfillment of each storage condition criteria:

Number of 'YES' responses

Total number of storage facilities surveyed

The total quantity and value of wasted medicines due to expire in 2011 Ethiopian fiscal year (July 8, 2018 to July 7, 2019) were evaluated. The medicines expired before July 2018 and accumulated over time were not considered. The beginning stock plus quantity received of the items during this year was evaluated and used as denominator.

Percentage of Stock Wasted due to Expiration

Unusable stock of an item during a period one year $* 100$

Beginning stock plus quantity received of the item during one year period

\section{Results}




\section{Background Information Of The Health Facilities}

Presence of Basic Infrastructures

Four hospitals and nineteen health centers found in west Wollega zone were surveyed, to evaluate warehouse management and reproductive health medicines expiration. Regarding the availability of basic infrastructures, $11(47.83 \%)$ of the surveyed health facilities had tarmac, $19(82.61 \%)$ had operational electricity, 16(69.57\%) had water and 22(95.65\%) had operational telephone during the study period (Table 1).

Table 1

Presence of basic infrastructures at public health facilities of west Wollega zone, Oromia region, Ethiopia, July 2019

\begin{tabular}{|c|c|c|c|c|c|c|c|}
\hline \multirow[b]{2}{*}{ S. No } & \multirow[b]{2}{*}{ Presence of Infrastructure } & \multicolumn{2}{|c|}{ Hospital(N = 4) } & \multicolumn{2}{|c|}{ Health centers $(\mathrm{N}=19)$} & \multicolumn{2}{|l|}{ Total $(\mathrm{N}=23)$} \\
\hline & & Yes & No & Yes & No & Yes & No \\
\hline 1 & Tarmac to the facility & $3(75 \%)$ & $1(25 \%)$ & $8(42.10 \%)$ & $11(57.90 \%)$ & $11(47.83 \%)$ & $12(52.17 \%)$ \\
\hline 2 & Operational Electricity & $4(100 \%)$ & $0(0 \%)$ & $15(78.95 \%)$ & $4(21.1 \%)$ & $19(82.61 \%)$ & $4(17.39 \%)$ \\
\hline 3 & Water & $4(100 \%)$ & $0(0 \%)$ & $12(63.16 \%)$ & $7(36.84 \%)$ & $16(69.57)$ & $7(30.43 \%)$ \\
\hline 4 & Operational telephone & $4(100 \%)$ & $0(0 \%)$ & $18(94.74 \%)$ & $1(5.26 \%)$ & $22(95.65 \%)$ & $1(4.35 \%)$ \\
\hline
\end{tabular}

In assessed health facilities, there were 77 health professionals with different professional backgrounds and educational levels under pharmacy units. From these professionals, $35(45.4 \%)$ were druggists. this research emphasized on store managers because they were principal personnel responsible for storage of pharmaceuticals. Majority (43.5\%) of the store managers were nurses and $18(78.3 \%)$ had taken formal IPLS training. Majority (39.1\%)) of them had 1 to 5 years of working experience (Table 2 ).

Table 2

Socio-demographic Characteristics of professionals working under pharmacy unit in Public health facilities of west Wollega Zone, Oromia region, Ethiopia, July 2019.

\begin{tabular}{|c|c|c|c|c|c|}
\hline S. No & Variables & & Hospital Frequency (\%) & Health Center Frequency (\%) & Total Frequency (\%) \\
\hline \multirow[t]{4}{*}{1} & \multirow[t]{4}{*}{ pharmacy unit staffing } & Pharmacist & $24(63.2 \%)$ & $3(7.7 \%)$ & $27(35.1 \%)$ \\
\hline & & Druggist & $14(36.8 \%)$ & $21(53.8 \%)$ & $35(45.4 \%)$ \\
\hline & & Nurse & $0(0 \%)$ & $14(35.9 \%)$ & $14(18.2 \%)$ \\
\hline & & Health Officer & $0(0 \%)$ & $1(2.6 \%)$ & $1(1.3 \%)$ \\
\hline \multirow[t]{3}{*}{2} & \multirow[t]{3}{*}{ Service year (store manager) } & $<1$ year & $1(25 \%)$ & $4(21.1 \%)$ & $5(21.7 \%)$ \\
\hline & & $1-5$ year & $1(25 \%)$ & $8(42.1 \%)$ & $9(39.1 \%)$ \\
\hline & & $>5$ year & $2(50 \%)$ & $7(36.3 \%)$ & $9(39.1 \%)$ \\
\hline \multirow[t]{3}{*}{3} & \multirow[t]{3}{*}{ Store managers trained } & IPLS & $4(100 \%)$ & $14(73.7 \%)$ & $18(78.3 \%)$ \\
\hline & & SC Overview & $2(50 \%)$ & $4(21.1 \%)$ & $6(26.1 \%)$ \\
\hline & & Never trained & $0(0 \%)$ & $5(26.3 \%)$ & $5((21.7 \%)$ \\
\hline
\end{tabular}

Availability equipment and furniture used in store

All hospitals had all basic equipment and furniture used in pharmaceutical store. However, majority of the health centers had no refrigerator, freezer, sufficient shelves, and office table with chairs (Table 3).

Table 3

availability of equipment and furniture in pharmaceutical store at public health facilities of west Wollega zone, Oromia region, Ethiopia,

\begin{tabular}{|c|c|c|c|c|c|c|c|}
\hline \multirow[t]{2}{*}{ S. No } & \multirow[t]{2}{*}{ Name of Equipment and furniture } & \multicolumn{2}{|c|}{ Hospital( $\mathrm{N}=4)$} & \multicolumn{2}{|c|}{ Health Center(N = 19) } & \multicolumn{2}{|l|}{ Total $(\mathrm{N}=23)$} \\
\hline & & Available & Not available & Available & Not available & Available & Not available \\
\hline 1 & Refrigerator & $4(100 \%)$ & $0(0 \%)$ & $6(31.58 \%)$ & $13(68.42 \%)$ & $10(43.48 \%)$ & $13(56.52 \%)$ \\
\hline 2 & Freezer & $4(100 \%)$ & $0(0 \%)$ & $6(31.58 \%)$ & $13(68.42 \%)$ & $10(43.48 \%)$ & $13(56.52 \%)$ \\
\hline 3 & Wooden pallet & $4(100 \%)$ & $0(0 \%)$ & $9(47.37 \%)$ & $10(52.63 \%)$ & $13(56.52 \%)$ & $10(43.48 \%)$ \\
\hline 4 & Sufficient Shelves & $4(100 \%)$ & $0(0 \%)$ & $7(36.84 \%)$ & $12(63.16 \%)$ & $11(47.83 \%)$ & $12(52.17 \%)$ \\
\hline 5 & Lockable cabinet & $4(100 \%)$ & $0(0 \%)$ & $15(78.95 \%)$ & $4(21.05 \%)$ & $19(82.61 \%)$ & $4(17.39 \%)$ \\
\hline 6 & Ladder & $4(100 \%)$ & $0(0 \%)$ & $7(36.84 \%)$ & $12(63.16 \%)$ & $11(47.83)$ & $12(52.17 \%)$ \\
\hline 8 & Computer & $4(100 \%)$ & $0(0 \%)$ & $8(42.11 \%)$ & $11(57.89 \%)$ & $12(52.17 \%)$ & $11(47.83 \%)$ \\
\hline 9 & Office table with 2 chairs & $4(100 \%)$ & $0(0 \%)$ & $13(68.42)$ & $6(31.58)$ & $17(73.91 \%)$ & $6(26.09 \%)$ \\
\hline
\end{tabular}

Page $4 / 12$ 
Manual LMIS forms like Bin cards, stock cards, receiving and issuing vouchers (model 19 and 22), Report and Resupply Form (RRF), Internal Facility Report and Resupply Forms (IFRRs) were available and being used by all health facilities. All assessed hospitals were using electronic-LMIS during the study period, while only $6(31.58 \%)$ of HCs were using electronic-LMIS (Table 4)

Table 4

Availability and utilization of LMIS tools in public health facilities of west Wollega Zone, Oromia regional state, Ethiopia, July 2019

\begin{tabular}{|lllllll|}
\hline LMIS Form & Available & \multicolumn{5}{c|}{ Utilized } \\
\cline { 2 - 7 } & Hospitals & HCs & Total & Hospitals & HCs & Total \\
\hline Bin cards & $4(100 \%)$ & $19(100 \%)$ & $23(100 \%)$ & $4(100 \%)$ & $19(100 \%)$ & $23(100 \%)$ \\
\hline Stock card & $4(100 \%)$ & $19(100 \%)$ & $23(100 \%)$ & $4(100 \%)$ & $19(100 \%)$ & $23(100 \%)$ \\
\hline e-Recording system & $4(100 \%)$ & $8(42.1 \%)$ & $12(52.17 \%)$ & $4(100 \%)$ & $6(31.58 \%)$ & $10(43.48 \%)$ \\
\hline Issuing voucher & $4(100 \%)$ & $19(100 \%)$ & $23(100 \%)$ & $4(100 \%)$ & $19(100 \%)$ & $23(100 \%)$ \\
\hline Receiving voucher & $4(100 \%)$ & $19(100 \%)$ & $23(100 \%)$ & $4(100 \%)$ & $19(100 \%)$ & $23(100 \%)$ \\
\hline RRF & $4(100 \%)$ & $19(100 \%)$ & $23(100 \%)$ & $4(100 \%)$ & $19(100 \%)$ & $23(100 \%)$ \\
\hline IFRR & $4(100 \%)$ & $19(100 \%)$ & $23(100 \%)$ & $4(100 \%)$ & $19(100 \%)$ & $23(100 \%)$ \\
\hline IPLS SOP Manual & $4(100 \%)$ & $19(100 \%)$ & $23(100 \%)$ & $4(100 \%)$ & $11(57.89 \%)$ & $15(65.22 \%)$ \\
\hline
\end{tabular}

\section{Storage Condition}

Among surveyed facilities, 17 (73.91\%) fulfilled desirable storage condition. When classified according to type of facility; all hospitals (100\%) and 13(68.42\%) health centers fulfilled desirable (>80\%) storage condition criteria (Fig. 1).

The storage condition criteria fulfilled by all the facilities include; stacking products at proper height, store products separately from chemicals and insecticides, protecting products from direct sun light (100\%), protecting cartoons from humidity (100\%), and securing storage area with lock and key (100\%). On the other hand, all the assessed facilities had no functional modern fire safety equipment. However, two (8.70\%) of health centers made local fire extinguishers from sand. In majority (52.17\%) of the facilities, the storage area is not sufficient (Table 5). 
Table 5

Number and percentage of warehouses that fulfill desirable storage condition criteria at public health facilities of West Wollega zone, Oromia region, Ethiopia, July, 2019(N=23)

\begin{tabular}{|c|c|c|c|}
\hline No & Description & Yes & No \\
\hline 01 & $\begin{array}{l}\text { Products that are ready for distribution are arranged so that identification labels and expiry dates and/or } \\
\text { manufacturing dates are visible. }\end{array}$ & 18(78.26\%) & $5(21.74 \%)$ \\
\hline 02 & $\begin{array}{l}\text { Products are stored and organized in a manner accessible for first-to-expire, first-out (FEFO) counting and general } \\
\text { management. }\end{array}$ & $18(78.26 \%)$ & $5(21.74 \%)$ \\
\hline 03 & $\begin{array}{l}\text { Cartons and products are in good condition, not crushed due to mishandling. If cartons are open, determine if products } \\
\text { are wet or cracked due to heat/radiation (fluorescent lights in the case of condoms, cartons right-side up for Depo- } \\
\text { Provera }{ }^{8} \text { ) }\end{array}$ & $23(100 \%)$ & $0(0 \%)$ \\
\hline 04 & $\begin{array}{l}\text { The facility makes it a practice to separate damaged and/or expired products from usable products and removes them } \\
\text { from inventory. }\end{array}$ & $18(78.26 \%)$ & $5(21.74 \%)$ \\
\hline 05 & Products are protected from direct sunlight. & $23(100 \%)$ & $0(0 \%)$ \\
\hline 06 & Cartons and products are protected from water and humidity. & $23(100 \%)$ & $0(0 \%)$ \\
\hline 07 & $\begin{array}{l}\text { Storage area is visually free from harmful insects and rodents. (Check the storage area for traces of bats and/or } \\
\text { rodents [droppings or insects].) }\end{array}$ & $18(78.26 \%)$ & $5(21.74 \%)$ \\
\hline 08 & $\begin{array}{l}\text { Storage area is secured with a lock and key, but is accessible during normal working hours; access is limited to } \\
\text { authorized personnel. }\end{array}$ & $23(100 \%)$ & $0(0 \%)$ \\
\hline 09 & Products are stored at the appropriate temperature according to product temperature specifications. & $20(86.96 \%)$ & $3(13.04 \%)$ \\
\hline 10 & Roof is maintained in good condition to avoid sunlight and water penetration. & $21(91.30 \%)$ & $2(8.70 \%)$ \\
\hline 11 & Storeroom is maintained in good condition (clean, all trash removed, sturdy shelves, organized boxes). & 18(78.26\%) & $5(21.74 \%)$ \\
\hline 12 & $\begin{array}{l}\text { The current space and organization are sufficient for existing products and reasonable expansion (i.e., receipt of } \\
\text { expected product deliveries for foreseeable future). }\end{array}$ & $11(47.83 \%)$ & $12(52.17 \%)$ \\
\hline 13 & $\begin{array}{l}\text { Fire safety equipment is available and accessible (any item identified as being used to promote fire safety was } \\
\text { considered). }\end{array}$ & $2(8.70 \%)$ & $21(91.30 \%)$ \\
\hline 14 & Products are stored separately from insecticides and chemicals. & $23(100 \%)$ & $0(0 \%)$ \\
\hline 15 & Products are stacked at least $10 \mathrm{~cm}$ off the floor. & 17(73.91\%) & $6(26.09 \%)$ \\
\hline 16 & Products are stacked at least $30 \mathrm{~cm}$ away from the walls and other stacks. & $20(86.96 \%)$ & $3(13.04 \%)$ \\
\hline 17 & Products are stacked no more than 2.5 meters high. & $23(100 \%)$ & $0(0 \%)$ \\
\hline
\end{tabular}

\section{Wastage Due To Expire And Proportion Of Expired Medicines}

A total value of reproductive health medicines lost due to expiration in all assessed facilities was 357,920.52(12,323.8 USD), 78,398 (2,699.4USD) in Hospitals and 279,522.52(9,624.4 USD) in assessed HCs. whereas, the total value of usable medicines was 4,093,961.2(140,962 USD). The Percentage of Stock Wasted due to Expiration was $8.04 \%$, and ranges from highest wastage rate for Levonorgestrel $0.75 \mathrm{mg}$ tablet $(33.08 \%)$ to the lowest wastage rate for combined oral contraceptive pills (3.51\%) (Table 6). 
Table 6

Value and percentage of reproductive health $(\mathrm{RH})$ medicines wasted due to expiration from July 8, 2018 to July 7, 2019 at public health facilities of West Wolle July 2019

\begin{tabular}{|c|c|c|c|c|c|c|c|c|}
\hline \multirow{2}{*}{$\begin{array}{l}\text { S. } \\
\text { No }\end{array}$} & \multirow[t]{2}{*}{ product Name } & \multicolumn{3}{|l|}{ Hospital } & \multicolumn{3}{|l|}{ Health Center } & \multirow{2}{*}{$\begin{array}{l}\text { Total } \\
\text { Usable }\end{array}$} \\
\hline & & Usable & Expired & \%age & Usable & Expired & \%age & \\
\hline 1 & Male Condom & 41310 & 0 & 0 & 118357.2 & 7265.2 & 5.78 & 159667.2 \\
\hline 2 & Etonorgestrel $68 \mathrm{mg}$ & 206829 & 5638 & 2.65 & 259516 & 66315.6 & 20.35 & 466345 \\
\hline 3 & IUCD & 70804.6 & 12192 & 14.69 & 495752 & 31526 & 5.98 & 566556.6 \\
\hline 4 & Jedelle & 55902.8 & 405 & 0.72 & 46383 & 3368 & 6.77 & 102285.8 \\
\hline 5 & Norgestrel $0.03 \mathrm{mg}$ & 7300.6 & 2983 & 29.01 & 158837 & 3565.8 & 2.20 & 166137.6 \\
\hline 6 & $\begin{array}{l}\text { Levonorgestrel } \\
0.75 \mathrm{mg}\end{array}$ & 5315.6 & 3100.8 & 36.84 & 9207.2 & 4079.6 & 30.70 & 14522.8 \\
\hline 7 & $\mathrm{COC}$ & 737329.8 & 3192.4 & 0.43 & 103696.6 & 27441.2 & 20.93 & 841026.4 \\
\hline 8 & Medroxyprogesterone & 33988.2 & 0 & 0 & 128456.8 & 12799.52 & 9.06 & 162445 \\
\hline 9 & Oxytocin $10 \mathrm{mg}$ inj. & 54108.8 & 1639.6 & 2.94 & 31168.4 & 3405.6 & 9.85 & 85277.2 \\
\hline 10 & Ringer Lactate & 71932.6 & 2530 & 3.40 & 25300 & 3520 & 12.21 & 97232.6 \\
\hline 11 & MgSO4 & 291072 & 13833.2 & 4.54 & 91932.8 & 6341.4 & 6.45 & 383004.8 \\
\hline 12 & Gentamycin & 6510.8 & 240.8 & 3.57 & 10836.2 & 1568.2 & 12.64 & 17347 \\
\hline 13 & Misoprostol & 189552 & 17952 & 8.65 & 83424 & 15376 & 15.56 & 272976 \\
\hline 14 & Ulcure kit & 3314 & 596.6 & 15.25 & 6628 & 1314 & 16.54 & 9942 \\
\hline 15 & Addis cure kit & 11658.8 & 0 & 0 & 8716.8 & 1794.2 & 17.07 & 20375.6 \\
\hline 16 & Addis cure plus kit & 19440 & 2643.8 & 11.97 & 20995.2 & 1676.4 & 7.39 & 40435.2 \\
\hline 17 & $\begin{array}{l}\text { Benzanthine } \\
\text { Penicillin }\end{array}$ & 2898.6 & 0 & 0 & 15420 & 5078.2 & 24.77 & 18318.6 \\
\hline 18 & Chlorhexidine & 4075.8 & 0 & 0 & 267618 & 31208 & 10.44 & 271693.8 \\
\hline 19 & Amoxicillin DT & 10831.6 & 1792.8 & 14.20 & 39964.6 & 3133.8 & 7.27 & 50796.2 \\
\hline 20 & Procaine penicillin & 1068 & 0 & 0 & 5340 & 1204 & 18.40 & 6408 \\
\hline 21 & ORS & 57112 & 726 & 1.262 & 147136 & 30564.6 & 17.20 & 204248 \\
\hline 22 & Zinc sulfate tab & 10163.6 & 8780.4 & 46.35 & 47101.6 & 10693 & 18.50 & 57265.2 \\
\hline 23 & TTC eye Ointment & 1365.4 & 151.6 & 10 & 5879.2 & 577.2 & 8.94 & 7244.6 \\
\hline 24 & Ceftriaxone & 19260 & 0 & 0 & 9630 & 1867 & 16.24 & 28890 \\
\hline \multirow[t]{2}{*}{25} & Vitamin A capsule & 6400 & 0 & 0 & 37120 & 3840 & 9.38 & 43520 \\
\hline & TOTAL & $\begin{array}{l}1,999,544.6(68,847.7 \\
\text { USD) }\end{array}$ & $\begin{array}{l}78,398 \\
(2699.4 \text { USD) }\end{array}$ & 3.92 & $\begin{array}{l}2,174,416.6(748688 \\
\text { USD) }\end{array}$ & $\begin{array}{l}279,522.52(9624.4 \\
\text { USD) }\end{array}$ & 11.39 & $\begin{array}{l}\text { 4,093,961.2(140962 } \\
\text { USD) }\end{array}$ \\
\hline
\end{tabular}

Regarding the contribution of each medicine to loss due to expiration, Etonorgestrel $68 \mathrm{mg}$ subdermal (implanon) contributed highest value, holding 71,953.6 ETB/2,477.49 USD (20.10\%) of expired medicines. Misoprostol with 17,952 ETB/618.12 USD (22.9\%), and Etonorgestrel 68 mg subdermal (Implanon) with 66,315.6 ETB/2,283.36 USD (23.72\%) contributed high value to loss due to expire in assessed hospitals and health centers respectively (Table 7). 
Table 7

Proportion of expired Reproductive health medicines at public health facilities of West Wollega Zone, Oromia region, Ethiopia, July 2019

\begin{tabular}{|c|c|c|c|c|c|c|c|}
\hline \multirow[t]{4}{*}{ S. No } & \multirow[t]{4}{*}{ Name of product } & \multicolumn{6}{|c|}{ Value and Proportion of each expired medicine } \\
\hline & & \multicolumn{2}{|l|}{ Hospital(N = 4) } & \multicolumn{2}{|l|}{$\mathrm{HC}(\mathrm{N}=19)$} & \multicolumn{2}{|l|}{ Total $(\mathrm{N}=\mathbf{2 3})$} \\
\hline & & Total Value (ETB) & Percentage (\%) & Total Value (ETB) & Percentage & Total Value (ETB) & Percentage \\
\hline & & & & & $(\%)$ & & $(\%)$ \\
\hline 1 & Etonorgestrel $68 \mathrm{mg}$ & 5638 & 7.19 & 66315.6 & 23.72 & 71953.6 & 20.1 \\
\hline 3 & Misoprostol & 17952 & 22.9 & 15376 & 5.5 & 33328 & 9.31 \\
\hline 4 & ORS & 726 & 0.93 & 30564.6 & 10.93 & 31290.6 & 8.74 \\
\hline 5 & Chlorhexidine & 0 & 0 & 31208 & 11.16 & 31208 & 8.72 \\
\hline 6 & Contraceptive pills & 3192.4 & 4.07 & 27441.2 & 9.82 & 30633.6 & 8.56 \\
\hline 10 & Male Condom & 0 & 0 & 7265.2 & 2.6 & 7265.2 & 2.03 \\
\hline 11 & Levonorgestrel $0.75 \mathrm{mg}$ & 3100.8 & 3.96 & 4079.6 & 1.46 & 7180.4 & 2.01 \\
\hline 12 & Norgestrel $0.03 \mathrm{mg}$ & 2983 & 3.8 & 3565.8 & 1.28 & 6548.8 & 1.83 \\
\hline 13 & Ringer Lactate & 2530 & 3.22 & 3520 & 1.26 & 6050 & 1.69 \\
\hline 14 & Benzanthine Penicillin & 0 & 0 & 5078.2 & 1.82 & 5078.2 & 1.42 \\
\hline 15 & Oxytocin 10 mg inj. & 1639.6 & 2.09 & 3405.6 & 1.22 & 5045.2 & 1.41 \\
\hline 16 & Amoxicillin DT & 1792.8 & 2.29 & 3133.8 & 1.12 & 4926.6 & 1.38 \\
\hline 22 & Gentamycin & 240.8 & 0.31 & 1568.2 & 0.56 & 1809 & 0.51 \\
\hline 23 & Addis cure kit & 0 & 0 & 1794.2 & 0.64 & 1794.2 & 0.5 \\
\hline 24 & Procaine penicillin & 0 & 0 & 1204 & 0.43 & 1204 & 0.34 \\
\hline 25 & TTC eye Ointment & 151.6 & 0.19 & 577.2 & 0.21 & 728.8 & 0.20 \\
\hline & Total & 78,398 & 100 & $279,522.52$ & 100 & $357,920.52$ & 100 \\
\hline
\end{tabular}

\section{Pharmaceutical store management challenges}

The challenges of warehouse mismanagement and factors contributing to wastage of reproductive health medicines were identified through in-depth face-toface interview with relevant key informants and the results were summarized under the following themes.

\section{i. Infrastructure Related}

Most of the KIs raised infrastructure related problem. Especially, most KIs from the HCs complained that the medical store was a simple room not built for the purpose of medical store. Especially, they explained these factors as a major reason for poor medicines storage. For example, one of the store managers explained the problem as follows:

As you see, the store is very congested. The roof is too short and it is difficult to clean daily. The roof needs maintenance. During rainy season, the water penetrates. I have reported to the management and they are discussing. It is long time since I told them. But they complain shortage of budget for maintenance.

In addition to the problem of the storeroom, KIs also complained lack of equipment and furniture used in store. Most of Kls from raised that lack of equipment like refrigerator, freezer, and insufficient shelves. This problem is stated by one of the Kls as follows:

We are putting cartoons on the ground due to lack of shelves. We have no refrigerator and freezer in our store. Therefore, we store cold chain products in another unit. Since I am not there, I do not know how much the temperature is and they do not record the temperature daily. Since there is frequent electric 
power interruption, the refrigerator there is not always functional.

\section{ii. Human power Related}

Another challenge identified by most KIs, especially in Health centers was, worker problem. They complained that due to insufficient pharmacy professional at their facility they assigned other professionals to manage pharmaceutical store. For example, one of the KIs expressed it as:

We have only one druggist in our facility. He does all pharmacy duties. We assigned him at dispensary. He also does procurement. So, we assigned midwife nurse at store and the druggist oversees it.

Another $\mathrm{KI}$ also stated:

I'm nurse. I have not taken any drug management related course during my college study, except pharmacology. However, due to lack of druggists in our facility, they assigned me as store manager. Most of the works are strange for me. I am just trying to manage all the works, but sometimes even, I encounter new terms. In addition, I have not taken any training.

\section{Factors Contributing to Drug Expire i. Supplier Related factors}

Most of the KIs were complaining the problem of the supplier. They stated that the supplier pushes medicines in bulk without their request.

Most of the time PSA and partners dumps a huge amount of family planning medicines with short expire date. We do not need most of these medicines. So, they expire in bulk at our facility.

Another $\mathrm{KI}$ also explained the problem as:

PSA sends near expiry drugs with very essential drug we need, to a driver. If we want to return those near expiry medicines, the driver does not allow us. He obliges us either to receive all products or return all the products. Since some products are very important, we cannot return them; we are obliged to receive those near expiry drugs.

\section{ii. Demand related}

Most KIs raised less customer demand for some family planning methods as contributing factor for expiration. One of the KIs explains this as;

Most of our customers prefers medroxy-progestrone (Depo-polivera) over other long-term methods. I think that could be due to its convenience for them. Since the supply from the partners is not need based, the bring other medicines with low demand. Then, those medicines will expire at our facility.

\section{Discussion}

\section{Availability of equipment and furniture}

Basic equipment and furniture are important for properly arranging, putting, and keeping medicines at specified temperature and humidity. It also facilitates first expiry first out (FEFO) principle. The current study revealed that all the hospitals had all basic equipment and furniture used in pharmaceutical store. However, majority of the health centers had no refrigerator, freezer, sufficient shelves, and office table with chairs. These results are better than the study conducted in East Hararge, Ethiopia(12). The probable reason for difference may be the study setting. The previous study was conducted in woreda stores, while the current is in hospitals and health centers. Lack of these equipment and furniture had contributed to poor storage management in health centers as, revealed by qualitative result.

\section{Storage Condition}

Warehouses used for storage of medicines should fulfill good storage condition criteria to keep the integrity and smooth flow of medicines. Among assessed facilities, $17(73.91 \%)$ fulfills $\geq 80 \%$ of acceptable storage conditions. This is slightly lower than the study conducted in Nigeria, where all facilities met acceptable storage conditions (13). The difference might be due to the difference in study setting. The previous study included central medical stores in addition to SDPs. But the current result is better than a similar study conducted by Gurmu and Ibrahim, which reported only $25 \%$ of the study facilities fulfilled the criteria of good storage condition ( $\geq 80$ positive response)(7). The difference may be because of some criteria like, practicing FEFO, depends on personnel engaged in the store management and were improved in the current study. Almost all the assessed facilities did not have fire safety equipment (91.30\%), even the left two had no modern fire safety equipment rather they made locally from sand, and in majority (52.17\%) of the facilities, the storage area was not sufficient. On the other hand, the storage condition criteria fulfilled by all the facilities include; stacking products at proper height, store products separately from chemicals and insecticides, protecting products from direct sun light (100\%), protecting cartoons from humidity (100\%), and securing storage area with lock and key $(100 \%)$. This finding is similar to the study conducted in Nigeria and in East Shewa( 7,13$)$. Even though most of the assessed facilities fulfilled desirable storage condition criteria, most of the facilities complain a challenge of poor warehouse infrastructure, administrative problems and shortage of professionals, as revealed by the qualitative method.

\section{Wastage due to expiration}

Reducing wastage of medicines saves the organization money and ensures customers receive quality medicines (14). In the present study, the total value of reproductive health medicines lost due to expiration in all assessed facilities was 357,920.52(12,323.8 USD), 78,398 (2,699.4USD) in Hospitals plus 
279,522.52(9,624.4 USD) in assessed HCs. The result was higher than the results of the study done by Gurmu and Ibrahim in east Shewa, Ethiopia(7). The difference could be because, the current study was done on program drugs, unlike the previous that was done on key essential medicines, which contains non program drugs. As revealed by qualitative result, program drugs were supplied in bulk. Again, the supply chain system for $\mathrm{MNCH}$ commodities (a major portion of RH medicines) is inconsistent and has not been integrated into the Integrated Pharmaceutical Logistics System (IPLS). Therefore, their supply does not need based(8).

The overall wastage rate due to expire was $8.04 \%$ (3.92\% for Hospital and 11.39\% for HCs). Wastage rate due to expiration ranges from the highest wastage rate for Levonorgestrel $0.75 \mathrm{mg}$ tablet (33.08\%) followed by Zinc sulfate $25 \mathrm{mg}$ dispersible tablet (25.38\%) to the lowest wastage rate for contraceptive pills (3.51\%). The result was higher than Ethiopian health sector plan for $2018 / 2019$ that is $2 \%(15)$. The probable reason for this high wastage rate might be receiving bulk and near expiry medicines, which had low customer demand, as revealed by qualitative method.

Since wastage rate in hospitals were lower than that of $\mathrm{HCs}$, it might be due to professionals' skills assigned in store management. All store managers are pharmacy professionals in hospitals and majority of them took training, while store managers are in HCs were non-pharmacy professionals and not trained. Limitation of the study

The study only included reproductive health medicines and health facilities. Pharmaceutical supply agency, the upper stream of supply chain was not included.

\section{Conclusion}

From this study, we concluded that pharmaceutical stores in hospitals had equipment and furniture, had desirable storage condition, and had less percentage of reproductive health medicines expired. Nevertheless, significant number of the health centers' medical stores do not comply with the recommended storage conditions, lack equipment and furniture, and had greater percentage of medicines expired. Infrastructure related, administrative, and human power related factors were identified as store management challenges. A major factor contributing for expiration was dumping huge amount of medicines with short expire date. So, the supplier should supply medicines based on their need, concerned government and non-government organizations try to avail equipment and furniture, and emphasis should be given to the impact of wastage of medicines due to expire.

\section{Abbreviations}

FEFO: First Expiry First Out; HC: health center; IFRR: Internal Facility Report and Resupply form; IPLS: Integrated Pharmaceutical Logistics System; KI: Key Informants; LIAT: Logistics Indicator Assessment Tool; LMIS: Logistics Management Information system; LSAT: Logistics System Assessment Tool; MNCH: Maternal, Neonatal, and Child Health; PHCU: Primary Health Care unit; RH: Reproductive Health; RRF: Report and Resupply Form; SOP: Standard Operating Procedures; USAID: United States Agency for International Development; USD: United States Dollar; WR: Wastage Rate

\section{Declarations}

\section{Ethics approval and consent to participate}

The investigators received ethical approval of the research from the ethical review board of Jimma University, Institute of Health. Letter of permission was also received from West Wollega Zonal health department. Data collectors clarified the objectives, benefits, and risk of the study to the participants and continued with the data collection after obtaining oral consent from participants. We excluded the name of participants to keep confidentiality.

\section{Consent for publication}

Not applicable.

\section{Availability of data and materials}

The data sets generated and/or analyzed during the present study are available from the corresponding author on reasonable request.

\section{Competing interests}

The authors declare that they have no competing interests.

\section{Funding}

Jimma University covered the data collection fees.

\section{Authors' contributions}

OK involved in reviewing articles, data collection process, involved in data analysis, interpreted data, participated in the sequence alignment and drafted the manuscript and communicated for publication. GT and DF also reviewed articles, participated in the design of the study and performed the statistical analysis, interpreted data and participated in the sequence alignment and drafted the manuscript. All authors read and approved the final manuscript.

\section{Acknowledgments}


The authors would like to thank Jimma University for facilitating the study. We are also grateful to West Wollega Zone health facilities for providing us all the necessary data and data collectors for their unreserved commitment.

\section{References}

1. EPFSA. The Ethiopian Pharmaceuticals Supply Chain Management System Monitoring and Evaluation Training Manual. 2014.

2. Shafaat K, Hussain A, Kumar B, Hasan R. AN OVERVIEW: Storage of pharmaceutical products an overview. 2013;(May 2014).

3. Balakrishnan Kokilam M, Ganesh Joshi, Veena Ganesh Kamath H. Assessment of Pharmaceutical Store and Inventory Management in Rural Public Health Facilities - A study with reference to Udupi District, Karnataka. Pharm Methods. 2015;6(2):53-9.

4. Daughton CG. Cradle-to-cradle stewardship of drugs for minimizing their environmental disposition while promoting human health. I. Rational for and avenues toward a green pharmacy. Environ Health Perspect. 2003;111(5):757-74.

5. MSH. Health system in action: Hand book for leaders and managers [Internet]. Management Sciences for Health. 2010. Available from: http://www.msh.org/resources/center/health-system-inaction.cfn.

6. Gebremariam ET, Gebregeorgise DT, Fenta TG. Factors contributing to medicines wastage in public health facilities of South West Shoa Zone, Oromia Regional State, Ethiopia : a qualitative study. 2019;1-7.

7. Gurmu TG, Ibrahim AJ. Inventory management performance of key essential medicines in health facilities of East Shewa Zone, Oromia Regional State, Ethiopia. Cukurova Med J. 2017;42(2):277-91.

8. Woinshet Nigatu, Abebe Bogale, Miraf Tesfaye, Masresha Assefa and FT. Maternal, newborn, and child health logistics system assessment, ethiopia. 2018;(May):1-70.

9. USAID | DELIVER PROJECT, Task Order 1. Logistics Indicators Assessment Tool (LIAT).Arlington, Va.: USAID | DELIVER PROJECT, Task Order 1. 2008;

10. USAID I DELIVER PROJECT TO 1. Logistics System Assessment Tool (LSAT). Arlington, Va USAID | Deliv Proj Task Order 1\r. 2009;(January).

11. EPFSA. The Ethiopian Pharmaceuticals Supply Chain Management System Monitoring and Evaluation Training Manual. 2014;(May).

12. Gizat Molla Kassie SM. Assessment of Pharmaceutical Store Management in Woreda Health Offices of West Hararge Zone. Int Res J Pharm. 2014;5(8):642-5.

13. Kolapo: Kolapo, Usman, Elizabeth Bunde ER and El. Nigeria: Contraceptive Logistics Management System Report. Arlington, Va.: USAID I DELIVER PROJECT, Task Order 1, 2009). Acad Manag J. 2009;(December).

14. Aronovich, Dana, Marie Tien, Ethan Collins, Adriano Sommarlatte and LA. Measuring Supply Chain Performance: Guide to Key Performance Indicators for Public Health Managers. Arlington, Va.: USAID I DELIVER PROJECT, Task Order 1. 2010;(May).

15. Federal Democratic Republic of Ethiopia Ministry of Health. National Reproductive Health Strategy 2016-2020. 2016;

\section{Figures}

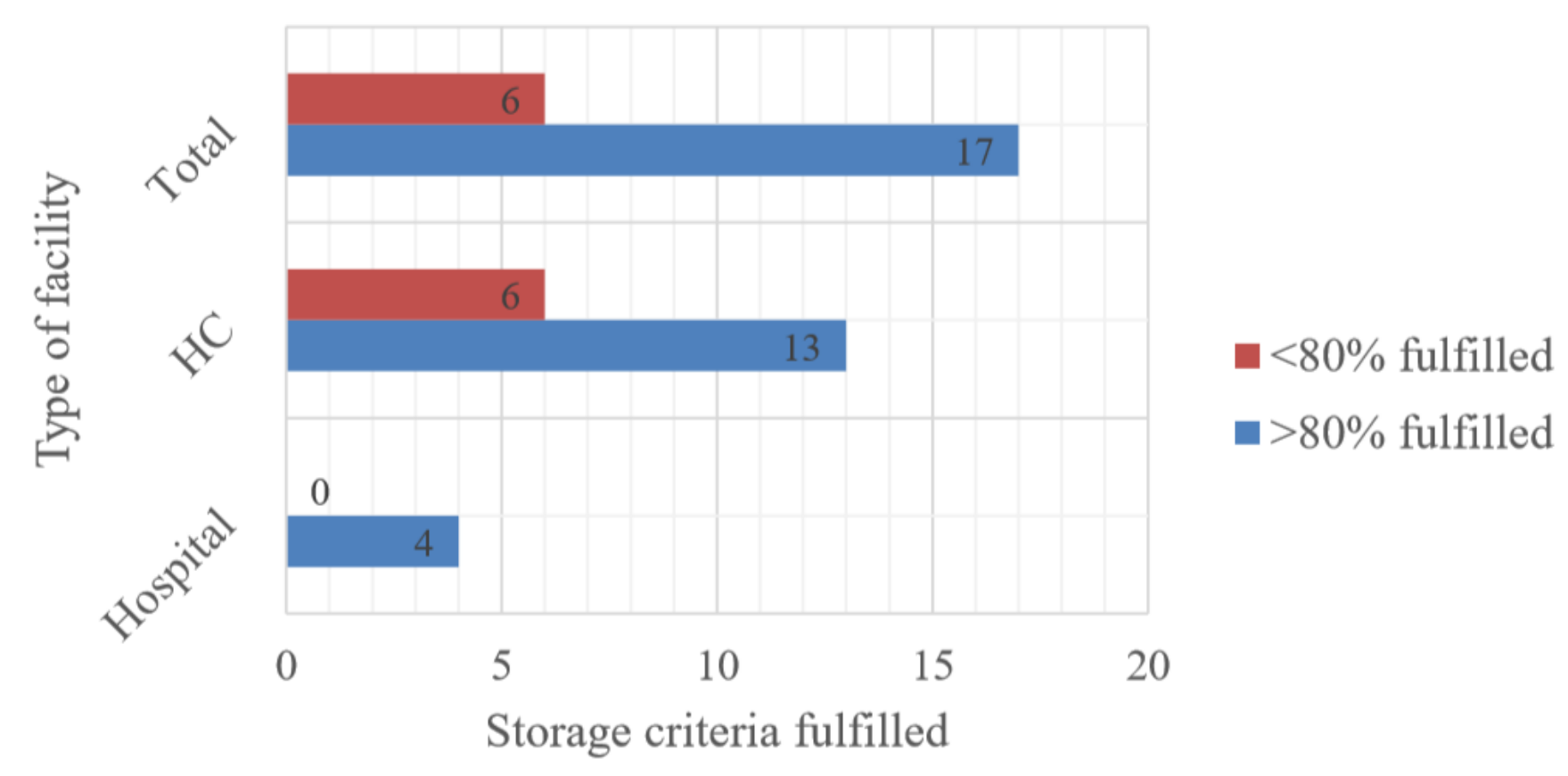

Figure 1

Page $11 / 12$ 
pharmaceutical stores fulfilling minimum good storage conditions criteria, at public health facilities of West Wollega zone, Oromia Region, Ethiopia, July 2019

Page $12 / 12$ 